Voix et Images

voixetimages

\title{
35 ans de vie littéraire : Yves Thériault se raconte
}

\section{Renald Bérubé}

Volume 5, numéro 2, hiver 1980

Yves Thériault

URI : https://id.erudit.org/iderudit/200203ar

DOI : https://doi.org/10.7202/200203ar

Aller au sommaire du numéro

Éditeur(s)

Les Presses de l'Université du Québec

ISSN

0318-9201 (imprimé)

1705-933X (numérique)

Découvrir la revue

Citer ce document

Bérubé, R. (1980). 35 ans de vie littéraire : Yves Thériault se raconte. Voix et Images, 5(2), 223-241. https://doi.org/10.7202/200203ar d'utilisation que vous pouvez consulter en ligne.

https://apropos.erudit.org/fr/usagers/politique-dutilisation/ 


\section{5 ans de vie littéraire: \\ Yves Thériault se raconte}

L'entrevue qu'on va lire est le résultat de trois séances d'enregistrement: les deux premières ont eu lieu à Rimouski, les 5 el 6 mars 1978, la troisième au domicile de l'auteur, le 19 aoüt 1979. En tout, quatre heures d'enregistrement dont le texte-montage qui suit essaie de donner un compte rendu aussi fidèle que possible. Mais comment rendre parfaitement les atmosphères multiples d'un entretien, d'une conversation, d'une discussion? Ainsi cette entrevue se déroule au " tu" ; nous avions pourtant convenu au départ d'utiliser le "vous" de circonstance. Mais nous l'avons perdu, it s'est échappé quelque part en cours de route et de discussion.

Cette entrevue, a sa façon, veut aussi être un témoignage. Voilà trois décennies et plus qu'Yves Thériault écrit et publie des livres, la parution des Contes pour un homme seul remontant à 1944 : il célèbre donc. celte année. le $35^{\circ}$ anniversaire d'une carrière littéraire plus que bien remplie. II vient aussi de recevoir, en cet automne 1979, te prix David; félicitations, donc, Yves Thériault. Mais surtout peut-être, bon anniversaire et longue vie, Monsieur Thériault.

Yves Thériault, qui a lu ce texte en octobre 1979, a fait le commentaire suivant: : Accord total, un total majuscule *.

\section{Renald BERUBE}

R.B. Si l'on essaie de retrouver les origines: d'où viennent les ancêtres d'Yves Thériault?

Y.T. A ma connaissance, pour autant que je sache, ce sont des Acadiens et des Indiens mélangés depuis deux ou trois générations avant moi. Mais ce sont surtout et avant tout des Acadiens. Mon père est né à SaintÉpiphane, puis il a vécu à Sainte-Rose-du-Dégelis, à Notre-Dame-duLac et à Cabano dans le Témiscouata. La famille de mon père a surtout vécu à Dégelis, en pratique mon père à été élevé là. Des Thériault, on en trouve beaucoup dans le coin: Notre-Dame-du-Lac, Edmunston - le haut de l'Acadie, il y a là un paquet de Thériault, tous de même souche, si tu veux. Mais j'en sais peu, vraiment peu. 
Le père de mon père est né à Saint-Épiphane. Ou à Saint-Bruno. SaintBruno. Oui. Mon grand-père Charles est déménagé de Saint-Bruno à Saint-Epiphane. Saint-Bruno de Témiscouata, pas Saint-Bruno de Montreal.

R.B. Et c'est le père de Charles qui aurait été indien?

Y.T. Oui.

A.B. Leur métier, qu'est-ce qu'ils faisaient ton grand-père et ton père?

Y.T. Des menuisiers. Its bâtissaient des granges et des églises. Ca se ressemble d'ailleurs, deux sortes de bâtisses semblables. C'était des spécialistes en granges et en églises.

R.B. Et toi, tu viens au monde à Québec?

Y.T. A Québec, en 1915. Mais ça, c'est une autre histoire. A un moment donné, mon pére est parti, il s'en est allé gagner de l'argent dans les villages miniers. C'était le début des mines d'amiante, Thetford, Black Lake, East Broughton. II s'est installé a Black Lake parce que... Je ne sais pas pourquoi, je ne l'ai jamais su. Et tout ce qu'il a trouvé comme travail : être commis au magasin général Hudon. La bâtisse du magasin général en question a d'ailleurs servi à recréer, parce qu'elle est toujours là, le magasin général du film Mon oncle Antoine. Mon père est arrivé à Black Lake, et il a eté commis là. Ma mère avait 26 ans. Elle a connu mon père, et mon père, il faut le dire, jeune, était un charmeur. C'étail un homme qui n'avalt pas beaucoup d'instruction, mais qui était beau, un bel homme, charmeur. Le père de ma mère, lui, était un prospecteur, un homme qui avait fait beaucoup de transactions, qui faisait affaire avec des Americains; il prospectait sur commande mais il vendait aussi des claims et participait a lorganisation de compagnies minières. Pas longtemps avant sa mort, il avait formé une compagnie a Cobalt, en Ontario, pour l'exploitation d'une mine qu'il avait découverte lui-méme, une mine de chrome et de nickel. Puis il est mort, il a paralysé, il a tout perdu. Mais avant cela, avant de paralyser, c'était un homme qui vivait à peu près - cela ressemble étrangement à son petit-fils - six fois au-dessus de son revenu. C'est de famille. C'est le monsieur qui possédait de trés beaux attelages, des chevaux splendides, qui possédait une terre à deux milles du village pour y garder ses chevaux. Black Lake et ses environs, a l'epoque, avait une composition démographique qui pouvait ressembler à Rawdon, ici, quant à la répartition anglophones-francophones. Bon. Ma mère menait donc une belle vie, mais le beau garçon qui s'appelait Alcide Thériault au magasin Hudon... Hudon et Ouellet... ils se sont épousés, et its ont eu deux enfants, Madeleine, ma sceur ainée, et moi.

R.B. La grande sceur est disparue tơt...

Y.T. Oui, elle est morte à 21 ans. II y avait, entre elle et moi, à peu près la méme différence d'àge qu'entre mes deux enfants, deux ans el demi environ. 
R.B. Né à Québec, mais pas longtemps après, la famille déménage à Montréal.

Y.T. Oui, c'est arrivé à peu près comme ceci. Alors que mes grands-parents maternels étaient en visite chez mes parents à leur logement de la rue du Pont - mon pere travaillait alors comme commis chez un gros épicier de Québec - mon grand-père a reçu un télégramme, de très mauvaises nouvelles. II se faisait poursuivre devant les tribunaux par un notaire de Black Lake pour libelle diffamatoire, mais le jugement n'était pas rendu encore. Pendant qu'il était a Québec, il a reçu un télégramme du jugement: if était coupable, avec quelque chose comme 60000 dollars à payer. II n'avait pas ce montant-la... II a paralyse, le choc, une thrombose cérébrale massive, comme j'en ai fait une, en 1970. Mais lui, il a paralysé complètement. Couché sur le dos, et seulement les yeux par lesquels on pouvait avoir une réponse à ce qu'on lui disait. II entendait, il comprenait, paraît-il, mais seuls ses yeux répondaient. Il est resté comme cela pendant deux ans, dans la maison de mon père, car mes grands-parents ne sont pas retournés à Black Lake. Je venais tout juste de venir au monde. Au bout de deux ans le grand-pere est mort, et puis alors mon père a décider d'aller s'installer à Montréal. Montréal, à l'époque, c'était l'endroit où il y avait du travail.

Mon père a repris son métier de menuisier, et la vie s'est déroulée comme dans la famille d'un homme qui travaille fort, trés fort, qui ne gagne pas beaucoup d'argent, car la fortune du menuisier est un phénomène très récent, qui date de quelques années seulement. Quand j'avais huit-dix ans, je me souviens, mon père était contremaitre et il gagnait trente dollars par semaine, quelque chose comme ca. Remarque qu'on vivait alors avec trente dollars par semaine comme aujourd'hui avec cent cinquante, à peu prés.

A Montréal, la première année. dont je ne me souviens pas d'ailleurs parce que j'étais trop jeune, nous avons habilé sur la rue de Lanaudière. De lá, et je m'en souviens parce que j'étais en âge de commencer l'école, on est allé habiter boulevard des Ormes, au Sault-au-Récollet, à Ahuntsic. Et puis, déménagement à Notre-Dame-de-Gráce. Le raisonnement de mon père était le suivant : it se fait beaucoup de construction a Notre-Dame-de-Grace. l'endroit était en pleine expansion, il y a plus de.chances de trouver du travail là que dans le nord de la ville. Il a eu raison, il a eu du travail. A NDG, nous avons habite sur Décarie, un endroit qui n'existe plus aujourd'hui, qui a été absorbé par les constructions, une petite maison situee à peu près en face du PrécieuxSang. Nous sommes restés là deux ou trois ans, et ensuite nous sommes allés habiter sur la rue Northcliffe. J'avais environ neut ans quand nous sommes partis de là, mon père venait d'acheter une épicerie à Drummondville. Puis it y a eu le retour à NDG, sur la rue Murat. puis à nouveau sur Décarie et Northcliffe. A NDG, nous avons toujours vécu dans un même cercle, disons, d'environ deux mille pieds autour de l'église. 
Puis, vers l'âge de quinze ans, j'ai abandonné l'école pour me lancer dans vingt métiers... Et ensuite il y a eu le sanatorium, puis la radio.

R.B. Yves Thériault a aussi piloté des avions...

Y.T. Oui, c'est un des métiers. Après le séjour au sanatorium. J'ai fait ce métier-là pendant deux ans; ce n'est pas beaucoup, je n'ai pas une vaste expérience. C'était en 1938 et en 1939. J'ai vraiment beaucoup aımé ce métier-là; et il y avait le plaisir de faire quelque chose que personne d'autres ne faisait, ou que bien peu laisait..

R.B. Le sèjour au sanatorium; l'expérience a été dure?

Y.T. Non... non, d'une cetaine façon j'ai même trouvé cela drôle. Ce qui a été dur... Vois-tu. j'avais fait de la boxe avant, et j'ai trouvé difficile d'admettre, d'accepter que je sois malade. C'est cela que j'ai trouvé dur... D'une certaine façon, je n'y croyais pas. Quand je suis entré au sanatorium - celui du Lac Edouard, en 1934 si mon souvenir est bon et que le médecin m'a vu, il n'y croyait pas lui non plus. Parce que tous les gars qui arrivaient par te train de nuit, le médecin les voyait le lendemain matin - et c'était tous des " pomoniques " qui se trainaient et qui toussaient. Moi je suis arrivé en pleine forme, en plein entrainement, j'avais fait du tennis, j'avais fait de la boxe: le médecin me regarde et me demande: "Qu'est-ce que vous faites ici, vous?", et je lui réponds: "Moi aussi je me le demande, je suis censé être tuberculeux". Il m'a examiné et il a trouvé, oui, que j'avais un voile au poumon, une infiltration calcaire au hile du poumon... je ne sais plus lequel d'ailleurs. Alors je me suis installé dans la routine du sanatorium; c'était suprèmement désagréable, tu étais obligé de te coucher dans l'après-midi et de faire ce qu'ils appelaient la cure silencieuse. Je n'avais pas du tout le goũt de me taire, j'avais bien plus le goût de blaguer. Au début c'était moins drōle; je voyais mourir les gens autour de moi... Mais j'avais beau faire, je me regardais dans le miroir le matin, et je me disais : “Voyons, je ne suis pas malade, moi... Je montais les escaliers en courant... Puis finalement je me suis mis a faire de la trappe avec des gars... j'en suis sorti.

R.B. Et en 1970, quand la maladie a trappé à nouveau, l'expérience a été plus dure?

Y.T. Non, pas plus dure. La mème... dureté, si tu veux.

R.B. La réaction en face de la maladie?

Y.T. Ca me met en colère... Tu trouves que cela n'a pas de sens, que c'est stupide... que les médecins devraient connaitre des... comme des solvants qu'ils pourraient l'envoyer dans les veines pour te debarrasser de ces saloperies-là. Bon, bon blague à part, je trouve bête que dans toutes les recherches on n'ait pas trouve une piqûre quelconque, une injection qui irait dans tes artères el qui dissoudrait ce qui ne doit pas 
être là. Tu sais ce dont tu souffres, mais en même temps tu ne peux rien faire, tu ne sais pas au juste ce qui t'arrive; je te dis, c'est choquant.

R.B. Écrire, au Québec, au début des années 1940, cela ressemblait à quoi ?

Y.T. Ca ressemblait à l'aventure de ceux qui, vers 1930 , sont montés en Abitibi défricher et qui ont mangé de la misère, misère physique, morale, financière. Il fallait qu'un cancer littéraire te ronge les tripes pour écrire au Québec à l'époque; il fallait avoir une vocation, une vocation profonde. Se préparer d'avance à crever de laim, parce qu'il n'y avait aucun barême commercial à l'époque. Les éditeurs étaient des amateurs, de profonds amateurs; les tirages étaient de 1000 ou 2000 exemplaires, le livre se vendait, disons deux dollars, ce qui était cher alors, et ton contrat te donnait au plus $10 \%$. Fais le compte, les chiffres étaient aberrants, même si le coût de la vie était bien bas. Tu travailles. tu travailles, mais c'est parce que tu as une vocation d'écrire, c'est parce que tu veux savoir si tu as quelque chose dans le ventre. Dans mon cas. les sous provenant des livres étaient un peu une dorure sur la tranche, parce que je gagnais ma vie à écrire déjà. j'écrivais les romans à dix sous, je faisais pas mal de radio - tout cela payait misérablement, mais j'en faisais beaucoup, et au bout du compte je réussissais à gagner autant, sinon plus, que le professionnel qui, dans son métier, avait le même temps d'expérience que moi dans le mien.

R.B. Dans ces conditions, autant sociales que personnelles, comment en vient-on à l'écriture?

Y.T. Paradoxalement, à travers tout cela, il y a une chose qui est toujours demeurée: je n'ai jamais aimé l'acte physique d'écrire. Peut-ètre parce que je suis un paresseux ; peut-étre surtout parce que je suis un gars qui est plus habile, linalement - l'aurail été, employons le conditionnel parce que je n'ai pas de preuve professionnelle de ce que j'avance aurait été plus habile de ses mains que la plupart des individus qui sont portés vers la chose intellectuelle. J'ai toujours regretté de ne pas avoir suivi un métier comme par exemple la menuiserie; je l'ai regretté, méme s'il n'y a pas de preuves que j'aurais eu là du succès.

R.B. Ton père était menuisier...

Y.T. Mon père était menuisier, mais il n'a jamais voulu que je continue le métier, il a toujours voulu que j'en sorte de ce métier-là.

L'acte d'écrire, l'acte de création est pour moi une torture, une torture dans le sens que je n'aime pas l'acte physique d'ecrire. Je suis extrémement heureux, étant jouisseur et l'ayant été toute ma vie, extrêmement heureux après, c'est-à-dire de constater le travail accompli. Je suis peut-étre mon premier lecteur totalement appro- 
bateur; je n'ai pas un esprit trop critique pour ce que je fais. Que cela ne m'ait pas nui m'étonne... mais je n'y peux rien. Ecrire, j'y suis venu presque par désespoir; parce que j'ai aussi une sainte et totale horreur du travail routinier, de la routine en général. De devoir me lever tous les matins, par n'importe quel temps, monter à bord d'un autobus, et aller travailler dans un bureau ou je serais aux ordres d'un patron qui aurait le privilège, un privilège qui n'est donné par Dieu ni par l'État, de me dire quoi faire, cela m'apparaissait comme un acte de masochisme gratuit. Et puis est arrivé un moment dans ma vie où il fallait que je travaille, et c'est tout à fait par hasard... et je raconte cela aujourd'hui avec une sorte d'émerveillement, en me disant combien le destin est d'abord et avant tout ce qui détermine ce qu'un homme sera, et presque pas sa propre volonté.

R.B. Le destin, c'est-à-dire?

Y.T. C'est-à-dire une petite annonce parue dans la Presse à la fin de 1945 au début de 1946 et qui se lisait ainsi : « Ecrivains demandès «, et donnait un numéro de téléphone. Je savais que j'avais probablement du talent, j'avais écrit et réalisé dèjà des petits dramatiques d'un quart d'heure pour un poste de radio qui jusque-là n'en diffusait pas du tout, $\mathrm{CKCH}$ à Hull; et auparavant j'avais aussi écrit un lot de contes qui avaient été publiés dans te Jour de Jean-Charles Harvey. Donc, je pensais que j'avais les qualités pour répondre à cette annonce-là; ce que j'ai fait, et le lendemain un monsieur d'une très belle prestance est venu me voir. II s'est nommé et m'a expliqué qu'il travaillait avec un éditeur qui publiait chaque semaine six romans à dix sous et un magazine qui s'appelait Police Journal. Magazine, fallait le dire vite, c'était un machin plié en deux avec une couverture de carton, quelque chose d'assez primaire. Le monsieur m'a demandé si je connaissais ces publications-là, je lui ai répondu que oui, je les avais vues dans les dépóts. II y avait les Aventures d'Albert Brien, détective, les Aventures extraordinaires de Guy Verchère, détective, il y avait aussi Diane, la belle aventuriere, de même que des romans d'amour. Entin, de toute taçon, ma femme, qui écrivait aussi, s'engage à faire trois romans d'amour par semaine, et moi trois detectives, Albert Brien, Guy Verchere, et un autre dont j'oublie le titre. Alors, j'ai commencè à écrire ces romans-là, et au bout d'un certain temps, puisque nous avions, ma femme et moi, acquis une méthode de travail extrêmement rapide, nous voulions en faire davantage. Nous sommes allés voir l'autre éditeur de romans à dix sous - ils étaient deux au Québec - qui lui aussi en publiait six, de son cóté. On a fini par écrire à peu près une douzaine de ces petits romans-là par semaine.

Je pense qu'il y a des gens dans la littèrature au Québec qui ne se vanteraient pas de cela, qui auraient trop d'orgueil pour s'en vanter. Pas moi. Pour la bonne raison que ce travail, de même que la pratique de la radio, m'a donné ce que je possède aujourd'hui el qu'un critique honnête va reconnaître, c'est-à-dire qu'il n'y a pas un écrivain québécois 
capable de construire un récil d'une façon aussi claire, nette et précise que Thériault. Aussi. cela m'a donné la faculté d'écrire très rapidement, aussi vite que la machine à écrire peut aller. Et là, on retrouve sans doute le fait que je n'aime pas l'acte physique d'écrire...

\section{R.B. Le milieu littéraire de ces années 1940 , à quoi ressemblait-il?}

Y.T. La chose assez extraordinaire. c'est que je n'ai pas vécu cette époque dans le milieu littéraire, je l'ai vécue complètement en dehors. Je faisais un texte de radio el une demi-douzaine a peu près de romans a dix sous par semaine; quand j'avais terminé cela, je n'avais pas le goût de m'intéresser à beaucoup de choses du mème genre... Oui, à l'époque je n'avais publié que les Contes pour un homme seul; les Conles avaient fait une belle marque, mais cela ne $m$ 'avait pas du tout amené dans le milieu littéraire. J'habitais assez loin de Montréal - parce qu'á l'époque. quarante milles de Montréal c'était loin. il n'y avait pas les routes d'aujourd'hui; j'habitais Saint-Denis sur le Richelieu, j'avais voulu, j'avais tenu a me réfugier là, parce que j'aimais la campagne et que j'abhorrais la ville; c'était un endroit paisible, sur le bord de la rivière. Mais ca me coupait des milieux littéraires.

\section{R.B. Et comment en arrive-t-on à la Fille laide?}

Y.T. La Fille laide, oui, ce roman a été l'entrée, si l'on veut, dans le milieu littéraire; le Marcheur aussi, qui est de la même année. On arrive à la Fille laide d'une curieuse façon, par un détour si l'on veut. En écrivant mes petits romans, j'avais acquis une telle vitesse qu'il me restait du temps libre, figurez-vous; et je suis un gars bilingue, elevé dans un quartier anglais, qui a beaucoup lu, qui lit toujours beaucoup en anglais. J'admirais beaucoup le travail qui se faisait du côté du short story en anglais; alors j'ai commencé à écrire des short stories en anglais. J'en ai écrit qui ont été publiés - ici, au Canada, une quarantaine ont été publiés à cette époque-là, dans différentes revues; et il y avait aussi une émission de short stories lues a CBC Toronto; d'ailleurs, à travers les années el jusqu'à tout récemment, je crois bien que j'ai ecrit une centaine de short stories pour CBC. Mais plus spécifiquement, quand je demeurais à Saint-Denis, i'ai commencé la partie préalable à une carrière de short story writer américain; c'est-a-dire que vous envoyez un texte avec aussi une enveloppe de retour dùment timbrée - et de 1944 à 1949 à peu près, j’ai dû envoyer je dirais une soixantaine d'histoires; ce que tu reçois évidemment, par retour du courrier, c'est un rejection slip, c'est-à-dire une note imprimée qu'on atlache au texle qu'on te renvoie. Mais, mais, à mesure que, dans le cas de certains magazines, je continuais, je persistais a envoyer des histoires; de plus en plus je commençais à recevoir des petites notes imprimées toujours, mais avec une phrase écrite à la main. Et en dernier, j'en étais rendu à recevoir des lettres, des lettres de refus, mais des lettres. C'étail déjà quelque chose d'extrêmement intéressant. Et dans deux cas, j'ai reçu une lettre me disant que j'étais passé à un poil... et une revue. Woird 
Tales, a acheté une histoire. Mais à ce moment-là, i'ai commis une erreur si je puis dire. Je ne vendais pas vite aux Etats-Unis, il fallait quand mème vendre pour pouvoir vivre, mon marché ici étail franchement français, le marché des romans à dix sous était tombé parce que l'agent qui servait de liaison entre l'éditeur et moi a été congédié par l'éditeur. J'ai opté á ce moment-là pour revenir à Montréal, j'ai abandonné mes tentatives du cóté américain, et j'ai choisi d'écrire surtout en français. Bien sür, je me disais que c'était une tâche fantastique que de réussir à gagner sa vie ici uniquement en écrivant... Mais j'ai décide ainsi, et j'ai laissé le marché américain. Parfois j'ai des regrets... mais c'est toujours facile d'avoir des regrets une trentaine d'annees plus tard...

R.B. Le mot, c'est en quelque sorte l'outil et le matériau de l'écrivain: pourtant l'on se méfie souvent du mot dans l'ceuvre de Thériault...

Y.T. Oui, mais l'ècrivain est aussi conscient de quelque chose d'autre que le mot, au-delà du mot, c'est-á-dire que le mot est finalement le soulignement d'un geste. Dans la langue, pour moi, les mots qui comptent vraiment ce sont les mots qui font l'action. Le mot est avant tout le transfert auditif d'un geste. II serait possible, par exemple, de ne jamais prononcer un mot. de remplacer tous les mots par des gestes. et la perception serait faite par l'œil plutôt que l'oreille. Je pense à ces émissions de télévision des prédicants américains où une personne traduit les paroles en gestes pour que les sourds comprennent. Donc, à la limite, et pourvu que l'on ait développé une série de gestes codifiés, de gestes qui racontent, on n'a pas besoin des mots.

R.B. Et tu aimes mieux le geste que le mot?

Y.T. Oui, parce que moi, ma communication passe d'abord et avant tout par le geste. Si tu m'observes dans une journée, tu vas te rendre compte que je précède, enchaîne et suis le mot très souvent pas le geste. Par l'altitude, le mouvement général du corps, le rythme du corps et le geste.

\section{A.B. Pourquoi continuer à écrire alors?}

Y.T. Parce qu'il y a des conventions qui existent. J'ai toujours pensè d'ailleurs que le cinéma était infiniment plus mon médium que la parole écrite, que le mot. Parce que le mot statique sur une page m'a toujours posé le problème de lutitisation d'un vocabulaire extrêmement fouillé, car il est fouillé mon vocabulaire, qui puisse arriver à transcrire pour l'œil ce que le geste, l'image, pourrait montrer infiniment mieux peutêtre. Cela a été quelque chose dans ma vie, le mot, parce que c'était finalement le seul moyen que j'avais. Je détestais le moyen - tout en 
adorant le mot, tout en vénérant le mot, je détestais la partie qui me restreignait dans le mot. C'est-à-dire que le mot ne devait, ne pouvait qu'évoquer une image, alors que j'aurais voulu pouvoir montrer, faire l'image. En écrivant, il m'arrive souvent de laire des gestes - c'est le geste qui vient, et c'est le mot que je cherche pour le traduire...

R.B. Mais malgré tout Yves Thériault a beaucoup écril; et s'il y avait les raisons matérielles évoquées tantôt, il y en avait d'autres aussi...

Y.T. Mais oui, mais oui il y en avait d'autres. Si je n'avais écril que pour des raisons matérielles d'ailleurs, étant donné ce que cela rapportait au Québec, j'aurais écrit bien davantage, pas une vingtaine, mais des vingtaines, plusieurs vingtaines de romans. II y avait d'autres raisons. Le dèfi, ici, de gagner sa vie par l'écriture, et de réussir cela en n'ayant qu'une huitième année d'école... Huitième année d'école, c'est-à-dire aucune des formations nécessaires pour devenir l'écrivain que je serais censé être, la certitude aussi que si javais eu ces formations-là je n'aurais pas èté l'écrivain que je suis... J'ai eu la sensation, un temps, d'une infériorité liée au manque d'instruction... mais il y avait aussi làdedans un jeu, un jeu destiné à me rassurer peut-être, et à rassurer les autres aussi. On vit dans un monde de conventions, et les conventions ont toujours exigé que l'être qui atteint à une réalisation quelconque ait passé par ce quion peut appeler les étapes, les étapes d'école. d'instruction, de connaissance. Il a élé influencé par ses maitres, il a acquis du savoir, et moyennant l'absorbtion de ce savoir-là, on lui a remis un certificat d'authenticité. Moi, je n'ai pas de parchemin dúment signé et contresigné, et on me l'a fait sentir et savoir d'une façon qui était parfois insultante. J'ai, dans mes cahiers de coupures de journaux, des articles qui disent à peu près ceci, que c'est bien beau ce que je fais, c'est beaucoup d'instinct, mais c'est de la merde dorée. Comment dire... ce que je fais n'est pas confirmed, sanctionné. Mais dit-on assez que chaque livre de Thériault est différent des autres, que personne ne lâchera un Thériault en cours de lecture? Bien sûr, j'ai pu écrire quelques ceuvres moins réussies, mais c'est normal... c'est comme dans une famille nombreuse, tous les enfants ne sont pas de même force.

Mais j'ai relevé le défi et j'ai joué le jeu, mème si on m'a dit que je ne pourrais pas y arriver, même si on me demandait pourquoi, avec ma formation, je me mèlais d'écrire... Les fois où la critique a été méchante envers moi, j'ai toujours joué le jeu - jeu, façon de parler, car j'étais aussi convaincu que mes livres pouvaient se défendre, bien sûr - de dire qu'est-ce que ca peut bien me faire la critique, mais cela m'a toujours fait profondément mal aussi... Le jeu, je parle de jouer le jeu... Tout écrivain, si académique soit-il, est un acteur, un conteur, un gars qui a besoin des applaudissements. Je l'ai déjá écrit. je suis aussi un cabotin, quelqu'un qui veut épater le citoyen; j'ai toujours cherché les applaudissements. je suis un gars qui a bâti une carrière d'écrivain sur le besoin. le besoin misérable d'avoir ces applaudissements. La vente des livres est le premier de ceux-ci, le fait d'ètre reconnu par un vaste 
public en est un deuxième. La renommée, l'approbation... mais je veux aussi plus que cela, je veux qu'on m'aime. Et quand, à la suite d'une rencontre avec le public, j'obtiens ce résultat-là, je suis, pour quelques heures, bien heureux.

Et puis, j'ai toujours été un conteur; étant conteur, je voulais raconter des histoires; et je voulais raconter des histoires parce que cela me faisail du bien de le faire. J'ai parlé de la torture d'écrire; mais il y a aussi l'envers de cela, la jouissance d'écrire. J'écrivais par besoin d'écrire, besoin viscéral d'écrire. Ne me demande pas des explications d'analyste universitaire, tout ce que je peux dire c'est que c'était plaisant de créer des personnages, de les faire vivre... Tu as un peu la sensation de jouer à Dieu, que tu es maître après Dieu, si tu veux... Tes personnages, tu as la liberté totale de les faire agir comme tu veux, où tu veux; el puis cela devient un défi, un défi psychologique, de pouvoir créer un personnage et de le faire agir selon ses normes à lui. De même, il y a tout le cóté structural - pendant que tu écris une phrase, devoir en même temps respecter une certaine unité structurale, prévoir quelle sera l'unité suivante ou la continuité de la première unité, ne pas faire faire au personnage des choses qui vont te créer des embètements selon la struclure que tu prévois pour l'ensemble. ne pas lui faire dire des choses, ou lui laire dire des choses qui vont emmener inexorablement telles conséquences... C'est un défi tout cela. Tout ce que tu lui fais dire ou faire, à ton personnage, doit ètre en fonction de son caractère, mais en fonction aussi des autres qui l'entourent, de ce que cela va provoquer chez eux et qui va aussi entrainer des conséquences dont tu devras te servir - et il faut que tout cela arrive juste, si je puis dire, car il y a une ligne dramatique, une unité que tu dois maintenir.

R.B. Mener ses personnages, mais une fois au monde ils ménent aussi.

Y.T. Oui, oui, ce qu'ils sont les mène et me mène. Si je crée une femme acariâtre, ce qui va s'ensuivre doit tenir compte du fait qu'elle est acariătre; donc. dans une certaine mesure elle me mène, elle mène mon inspiration, ma structuration des choses.

R.B. Tu as déjà fait remarquer que, en régle générale, il y a deux ou trois personnages importants dans tes romans, jamais beaucoup plus. C'est une caractéristique du conteur?

Y.T. Le conteur, en somme, raconte un instant dans la vie des gens. Ce qui explique une autre caractéristique de mes romans : rares sont ceux qui impliquent une longue continuité de vie. Il va y avoir des retours en arrière, à un moment donnè, pour justifier le personnage ou ses agissements; mais rarement $y$ a-t-il dans mes romans, une longue durée entre le point de départ el le point d'arrivée. Sur ce plan-là, Pierre Huneau constitue une exception; mais gènéralement, dans le Dernier Havre par exemple, méme si le personnage est âgé et même s'il y a des retours en arrière, l'épisode de sa vie qui me concerne est un épisode 
court, qui arrive assez rapidement à sa conclusion. De même, Agaguk ne raconte pas la vie d'Agaguk: la durée du roman est de cinq ou six ans, le temps pour le couple d'avoir deux enfants, d'avoir deux fois des enfants, plutôt. Et je lâche mes personnages quand cela fait mon affaire; quand le punch, quand la chute est là, alors c'est tout, c'est fini.

R.B. L'ordonnance générale, la structure de l'œuvre est très importante?

Y.T. Ah! mais oui. Si tu veux que ton lecteur soit pris dès les premières pages et ne láche pas jusqu'à la dernière, il faut que ton organisation structurale, matérielle de l'œuvre soit très très précise. Autrement tu le perds, tu le perds parce que toi-même tu te perds. J'ai entrepris la lecture de certains romans de ces dernières années et j'ai lâché en cours de route; sans doute l'auteur se comprenait-il, mais moi je ne m'y comprenais pas.

R.B. Le rapport entre le personnage et la structure: l'un des éléments est plus important que l'autre? ou est-ce que les deux sont liés?

Y.T. Les deux sont liès, sans priorité d'importance. Si le personnage est fort. s'il se tient, c'est quand même déjà assez concret comme élément de structure; que j'ajoule à cela une structure matérielle qui elle aussi soit très concrète, eh bien les deux s'interpénètrent sans que tu puisses dire que l'un est plus important que l'autre. Je ne pense pas qu'il soit possible, je n'ai jamais essayé de prendre un personnage faible et de le structurer dans un récit-qui serait fort. A ce moment-là je rate mon coup... C'est peut-étre le cas dans N'Tsuk où le personnage n'est pas vraiment très fort.

R.B. On établit souvent une distinction entre. d'une part, les écrivains qui sont tres conscients de ce qu'ils veulent faire, qui savent d'où ils partent et où ils veulent arriver, et, d'autre part, les écrivains plus inconscients, dont l'œuvre est comme un surgissement si l'on veut; par rapport à ces deux póles, ou Yves Thériault se situerait-il ? Plutót du cóté de l’écrivain inconscient, ou plutôt du cóté de celui qui organise?

Y.T. Du cóté de celui qui organise. Qui organise sans paraitre organiser c'est cela l'important. J'organise sans avoir l'air d'organiser. Tu a lu le début d'un texte de radio que j'ai commencé... Moi, j'organise à mesure. II y a quelque chose de très subconscient derrière qui dit "ton chemin c'est ça, ça devrait être ça\%. Comment puis-je y arriver? Dans ce texte de radio, quand j'ai écrit le premier paragraphe, je ne savais pas trop trop à quoi cela pourrait mener.

R.B. Ce paragraphe qui décrit la montagne...

Y.T. Oui. Un peu plus loin، il m'est venu dans l'idée que ce serait inléressant si l'homme ou la femme se réveillait la nuit et entendait de vastes bruits sourds et des grondements dans la montagne. Je ne savais pas ce que seraient ces grondements. Alors j'ai continué, et maintenant je sais ce que sont ces grondements. 
R.B. Ils se sont expliqués à mesure que...

Y.T. Pendant que j'écrivais, je me posais la question à savoir quels étaient au juste ces grondements, comment ils pourraient devenir une tragédie pour les gens: à mesure que je me posais ces questions, et tout en écrivant, jai commencé à savoir... Quand tu vas lire le texte ou que tu vas l'entendre a la radio, il va te paraitre très structuré. Il est évident que j'aurais pu arriver à la mẽme structuration en me donnant au départ un plan très précis, détaillè - mais qui est froid. J'aime mieux organiser mon plan à mesure que je fais le texte. La structuration d'instinct. si tu veux, me mène vers la structuration délibérée. A peu près tous mes romans ont été écrits de cette façon; Agoak est une exception dans la mesure où je m'étais donné un plan assez détaillé avant de l'écrire. J'ai toujours travaillé avec un plan qui se formulait à mesure; rendu à la moitié du roman à peu près, tu sais quelle sera ta finale - tu sais alors pourquoi, inexorablement, le roman doit s'en aller dans cette directionlà. Toul ce que tu as écrit jusque-là te mène là; la petite lueur. ce que tu savais comme subconsciemment et qui te disait que c'était probablement dans telle direction que tu devais aller mais ne te disait comment tu pourrais te rendre au point pressenti, la petite lueur devient clarté ; vers le milieu du roman tu sais comment tu vas t'y rendre, parce que tu es en train de t'y rendre, c'est fait.

R.B. Prenons un exemple pour illustrer tout cela, Moi, Pierre Huneau disons.

Y.T. Dans le cas de Pierre Huneau, il y a eu du travail de recherche avant la rédaction de l'œuvre; j'avais le désir intense d'écrire quelque chose dans une langue précise, celle d'un pècheur gaspésien né dans la deuxième moitié du siècle dernier. Pour bien posséder celte langue, il m'a fallu fouiller, jouer du dictionnaire, vérifier des mots, des expressions. J'ai mis trois mois à écrire Pierre Huneau, une centaine d'heures, ce qui est beaucoup pour moi... Non, dans le cas de Pierre Huneau il n'y avait pas de structure antérieure à l'œuvre, mais énormément d'instinct du personnage... je lui ai donnè un rythme, une façon d'être, inévitablement il fallait qu'il suive un certain chemin. Bon, j'en ai fait un pécheur en Gaspésie ; à partir du moment où il était pêcheur en Gaspésie, il avail des préoccupations, des obligations, un destin propre. des gestes quotidiens, une vie. C'était moi qui lui donnais le privilège d'être pêcheur, mais c'était lui qui, ensuite, suivait sa vie de pècheur. tout en ayant derrière lui le gars Thériault qui se dit à un moment donné : bon, c'est logique à ce moment-ci, pour que le récit ait certains rebondissements, qu'il $y$ ail un naufrage. Un naufrage... Huneau, comment tu fais naulrage quand tu es pècheur et pourquoi? Huneau dit "je fais naufrage quand je suis pêcheur parce que j'ai commis telle erreur ou qu'il s'est produit tel événement, et ma barque s'est écrasée". Le personnage se mène dans la mesure où dans son mètier, sa profession, son "roule" de vie, il soit logique, coherent qu'il lui arrive telle ou telle chose; ensuite, c'est à moi de voir à les lui faire arriver avec une certaıne èlégance. 
A.B. La technique est importante, mais $Y$ ves Thériault ecrit avec ses tripes, selon l'expression souvent employée.

Y.T. Oui. Ecrire avec ses tripes, cela veut dire simplement que ton personnage et l'histoire qui en découle sont des choses que tu ressens profondément en toi, et que tu ne te laisses par arrêter par des considérations... par les espèces de théorisation quapportent certains professeurs, certains critiques. Ceux qui parlent de litterature font souvent de celle-ci quelque chose de très folklorique, au sens vèritable du mot folklore. C'est-à-dire que pour ces gens-là, un écrivain est un individu qui, derrière un grand pupitre, avec une plume d'oie, trace péniblement, sur des parchemins anciens, des mots immortels; et il écrit non pas avec ses sensations humaines, physiques, mais avec ses pensées, ses hautes pensées. Eh bien moi je ne travaille pas comme cela; je travaille avec une machine à écrire, et if m'est arrivé en travaillant de pleurer, de brailler parce que c'était émouvant ce que j'écrivais... c'est cela écrire avec ses tripes. Avoir faim avec ses personnages, avoir froid avec eux...

R.B. Quand l'cuvre est achevée et qu'on en sort, on en sort vide?

Y.T. Épuisé physiquement. Ashini - quand j'ai écrit Ashini par exemple, le temps que j'ai mis à l'écrire, quelques jours, je n'ai pas dormi durant ces jours-là. L'épuisement physique est tel que même le sommeil n'est pas possible, tellement on est au-dessus, si tu veux, du besoin de sommeil.

R.8. Les personnages, dans différents romans de Thériault, se méfient souvent de l'entourage...

Y.T. Je comprends... Tu ne te méfies pas de l'entourage, toi ? C'est la chose la plus normale du monde; se fier à l'entourage, mais c'est le suicide... Se fier aveuglèment à l'entourage? Faut pas... La tribu humaine est hostile; en d'autres mots, la tribu humaine n'est pas nécessairement consacrée au bien-ètre de la tribu. Elle n'est surtout pas occupée au bien-ètre de l'individu. Elle est occupée individuellement à son bienêtre à elle; si tu viens contrecarrer son bien-être d'une façon ou d'une autre, elle va y voir. Transpose cela, place cela dans la perspective du groupe villageois, du groupe citadin, du groupe provincial, du groupe fédéral, regarde tout ce qui se passe, jette un coup d'œil sur l'ensemble des choses, et demande-toi si, aveuglément, tu dois te fier à ton entourage. Non. Je me fie, mais pas aveuglément.

R.B. Ce qui explique que l'individu, dans les romans de Thériault, a souvent beaucoup de difficultés avec la collectivité? 
Y.T. Oui. Il en est ainsi dans mes romans parce que dans la réalité les choses se passent ainsi. Tu vas voir comment par exemple dans un village, tu le vois d'ailleurs beaucoup mieux dans un village que dans une ville. comment dans un village l'on va s'acharner à détruire quelqu'un. Et ça, c'est l'histoire du monde. Je regardais une émission sur Moïse l'autre jour à la télévision; vois ce qui est arrivé quand Moise s'est absenté. Quand il est revenu, les siens adoraient le veau d'or; alors lui, comme homme, il a dû retaire son emprise sur eux. Déjà les siens étaient prêts à détruire Moise. Le symbole est fantastique. il est dans la Bible et il s'applique encore aujourd'hui.

R.B. As-tu èté un lecteur de la Bible?

Y.T. Oui, à un moment donné je lisais beaucoup la Bible, c'ètait presque un livre de chevet. Dans les années quarante; je ne la lisais pas par catholicisme, mais parce qu'elle m'intéressait.

A.B. L'entourage, ce peut être les hommes, ce peut être les éléments naturels aussi, la mer par exemple, dont parle le vieux narrateur rusé du Dernier Havre.

Y.T. Le personnage du Dernier Havre vit d'une part dans son univers présent, mais il nous raconte aussi son univers antérieur qui, prétend-il, a èté bien serein, mais ce n'est pas vrai; un pécheur en Gaspésie connait aussi des menaces - il y a ses acheteurs de poissons, il y a la mer qui est un danger, il y a sa Caisse populaire dont les gens ne reprèsentent peut-ètre pas un danger, mais peuvent être négatifs à un moment donné à son égard. En tant que pêcheur, il est passé à travers une vie durant laquelle il a dū ruser passablement; d'abord pour prendre le poisson, ce qui demande une certaine ruse, ensuite pour conquérir la mer, ce qui demande une certaine ruse aussi parce qu'elle est traîtresse...

R.B. C'est une vieille fascination la mer...

Y.T. Elle est vivante la mer, elle est vivante. La mer peut procurer une vie à l'homme, elle peut le nourrir, c'est une amie, c'est une ennemie, c'est quelque chose de grand. d'immense; il n'y a pas un homme qui a conquis la mer. On peut dire. ah! tel decouvreur qui dans les premiers temps s'est promené dans une nef fragile a conquis la mer d'un continent à l'autre; mais ce n'est pas vrai, il n'a pas conquis ta mer, il s'est laissé flotter dessus et les choses ont bien tourné.

En fait la mer, la forèt. l'Arctique, le désert du Sahara, les montagnes Aocheuses, les Alpes, la Cordillere des Andes, tout cela c'est la même chose; ce sont des entités de nature où un homme peut trouver et la mort et la vie. C'est un défi pour l'homme. La mer est un défi parce que personne ne peut prendre la mer dans un bateau ou une barque et se vanter de la franchir toute sans que jamais la mer ne veuille avaler la barque. Et la montagne je peux la franchir, mais je ne sais 
jamais quand il y aura un précipice pour me faire dégringoler; le désert je peux y mourir de soif...

Revenons au personnage du Dernier Havre. II est vieux maintenant, et personne ne veut plus de lui; tant qu'il était pêcheur, qu'il ramenait sa morue, qu'il avait toutes ses forces, tout allait assez bien. La société qui le délaisse, les forces qui diminuent, deux sortes de menaces, si lu veux... les menaces que ressent chacun sont à la dimension de chacun, s'accordent à la situation de chacun. Menaces, défis en même temps; la fin du Dernier Havre, c'est un triomphe pour le vieux, un triomphe contre la socièté, il va chercher une mort, sa mort, pas la mort imposée, mais sa mort qu'il a faite lui-même. Quand il se laisse couler dans sa barque, quand il fait volontairement couler la barque dans laquelle il se trouve, il va chercher la vraie sérénité, celle de la mort.

R.B. Le décor, le lieu de l'action, est donc très important. Qu'il s'agisse de la montagne, par exemple, de la toundra...

Y.T. On peut préférer la montagne, les rondeurs de la montagne, à l'étendue plate de la plaine, on peut prétérer le fleuve, l'eau, la forêt... La montagne a de multiples couleurs, de multiples formes; tu regardes l'eau, surtout l'eau d'un fleuve comme le Saint-Laurent, encore là il y a toutes les couleurs, toutes les formes... Quand j'écris, j'ai la mème façon de voir les choses qu'un peintre; j'ai écrit sur les Esquimaux et la toundra, sur l'Ouest et ses bestiaux, sur les goélettes dans le fleuve, sur un individu qui était barbier dans un village... pour moi le sujet fait son décor ou le décor fait son sujet. Je n'ai pas d'attachement particulier ou à la montagne ou a la plaine; autant que possible, je vais essayer de travailler à partir de décors que je connais, la Cóte Nord par exemple.

R.B. De mếme qu'il y a une opposition individu-collectivité dans ton ceuvre, il y a aussi une opposition écologie-technologie, pour employer un langage courant aujourd'hui; on retrouve cette opposition-là dans la Passe-au-Crachin par exemple.

Y.T. II peut y avoir là un paradoxe. La technologie me fascine; il y a une chose qui me fascine plus que toute autre et qui est tellement loin des préoccupations habituelles de l'écrivain que j'ai presque honte de l'avouer devant certaines gens: le développement extrêmement fascinant, ces dernières annèes, dans le monde des ordinateurs. L'ordinateur, celle grosse, celte immense bête, cette énorme bête technologique qui est devenue, grâce à une découverte récente, une bête qui n'est plus énorme du tout et qui se terre dans une boite pas plus grosse qu'un étui de machine à écrire. Ce petit espace, cette petite boite peut contenir 25000 facteurs mémoriels, et voila qu'on peut la tenir entre ses deux mains. Elle coùte assez peu maintenant, et peut accomplir des tâches absolument incroyables. Je suis fasciné par le fait que l'on peut installer dans un si petit espace tout ce qui était dans le monstre originel 
et lui farre accomplir encore plus de choses quau premier. Faire déterminer, faire diriger, faire déclencher, faire dominer... le mot puissance, oui... Mais tout ne peut être dominé par une machine; c'est beau que la machine puisse accomplir des choses, et dans ce qui vient de vie, dans le siècle ou/el les siècles qui s'en viennent, on va assister à d'autres développements de ces puissances - et la seule survie possible de l'homme sera d'être capable de dominer la machine. La façon la plus primitive, la plus èlémentaire, presque simpliste de le faire, c'est celle d'Agoak; mais elle présage d'autres façons que l'homme aura de dominer la puissance.

Fasciné par la technologie, mais absolument débâti par le fait qu'on construise des centrales nucleaires. Avec cela je ne suis pas d'accord. Pour moi, il est trés important que la nature, ce qui est lout, l'eau, la montagne, les arbres, les bētes, que la nature survive: c'est pour cela que tu trouves toujours dans mon ceuvre des personnages extrêmement attachés à la nature, pas dans le sens qu'ils diraient "il faut protéger les bêtes ", mais attachès d'une façon totale à la nature, et même issus de la nature. Dans toule mon ceuvre, c'est lá un fil conducteur.

R.B. Préserver la nature, tout en sachant qu'on ne peut èvacuer tout ce que la technologie apporte.

1.T. On sait qu'on ne peut le faire, mais si on pouvait donc, tu vois... Peutêtre que ma fascination pour les énergies parallèles provient du fait que je ne voudrais pas qu'on détruise la nature au nom de l'énergie. Dans tout ce que j'ai écrit, toujours la nature triomphe, ou ne soutfre pas de la technologie. El il ne faudrait pas qu'elle en souffre. Donc, il y a refus de la technologie, la nature est prépondèrante. Mais la technologie nous échappe aussi, elle échappe à tout le monde, le progrès...

A.B. A la fin des Temps du carcajou, il y a ce moment où Juchereau affronte la nature, le coup de chien du Golfe...

Y.T. Juchereau a èté soudainement placè devant un défi subit, instantanè, et il a triomphé.

R.B. Mais après, le remords commence - il n'est pas très heureux Juchereau...

Y.T. Mais tous les triomphes ne sont pas heureux, rares sont les triomphes totalement heureux.

A.B. Agaguk et le grand loup blanc - pourquoi, qui est, si je puis dire, ce loup?

Y.T. J'avais besoin de faire défigurer Agaguk. Ce n'était pas possible qu'il soit défıguré par un ours blanc, qui est en somme la bête standard de l'Arctique, parce qu'un ours blanc pèse 2,000 livres et plus; el une bête d'une tonne qui lève une grosse patte qui pèse elle-mème 150 livres 
pour la rabattre dans le visage d'un homme - il n'y a plus de visage d'homme, il n'y a même plus d'homme, tout est labouré, déchiré par les griffes. J'avais besoin d'une défiguration d'Agaguk qui puisse empêcher qu'on l'identifie, mais il fallait aussi qu'Agaguk puisse continuer à vivre d'une laçon relativement normale, qu'il ait ses yeux... Le seul animal qui pouvait faire cela dans l'Arctique, c'était un loup, un jeune loup préférablement, qui ne pèserait pas plus de 75 livres; comme le loup. spontanément, n'attaque pas l'homme, il fallait que ce soit un loup lou. La seule bête folle, c'est la bête albinos, parce qu'elle est déséquilibrèe. elle ne se comporte pas selon les normes. Alors j'ai imaginé un loup blanc. Plus tard on en a fait un grand symbole; franchement, je n'ai pas pensé au symbole du tout, j'ai pensé à la sorte de bête qui pourrait me défigurer Agaguk tout en me permeltant de le récupérer ensuite pour le reste de l'hisloire. C'est aussi simple que cela. Le loup blanc n'est pas le symbole de l'homme blanc dans l'Arctique, je n'ai même pas pensé à cela.

R.B. Après trente-cinq ans d'écriture, comment Yves Thériault voit-il son ceuvre?

Y.T. Abondante... J'ai fait ce que je voulais faire, j'ai réussi ce que je voulais réussir. Ce qui est important pour moi, c'est la variété de mon œuvre, variété des cadres, variété des personnages. Beaucoup d'écrivains n'ont toujours écrit que le mème livre tout en écrivant plusieurs livres, ou n'ont toujours raconté qu'une histoire, la leur. J'ai voulu pour ma part m'attarder aux autres, inventer d'autres personnages que moi-mème, inventer de nouveaux livres chaque fois que je publiais. Si lu prends tout ce que j'ai écrit, tu peux peut-être trouver un lien d'un livre à l'autre, mais je n'ai jamais récrit le même livre. Prends lo Grand Roman d'un petit homme et le Dernier Havre par exemple; les deux personnages sont complètement différents, ils n'ont pas subi les mêmes frustrations du lout, ils n'ont pas été placés dans le mème cadre de vie, n'ont pas eu les mêmes ambitions ni les mêmes rêves. Fais le tour: comment rapprocher Aaron et les Temps du carcajou?

J'ai quittè l'école en huitième année; on m'a dil que je n'arriverais à rien dans l'écriture. Un défi. Et pourtant, aujourd'hui, bien peu d'éditeurs au Québec refuseraient un manuscrit de Thériault - et je ne me vante pas en disant cela, je mesure le chemin parcouru, je souligne le travail que cela signifie. Et pas seulement du travail d'ècriture; je n'ai jamais hésité, par exemple, à rencontrer souvent le public dans des entretiens, à faire du travail de relations publiques pour la vente de mes livres. Je l'ai fait en toute lucidité, en me disant qu'il fallait le faire.

R.B. Yves Thériault est un homme serein? 
Y.T. Raisonnablement serein. J'ai des tourments, on en a tous. Dans l'ensemble, assez serein. Peut-être que j'ai toujours été finalement assez serein, dans l'ensemble aussi. II y a des choses inexorables, il y a des choses inéluctables... Plus jeune j'avais la sensation qu'il n'y avait d'inexorable que la mort et d'inéluctable que ce que tu rendais inéluctable, parce que tu pouvais toujours combattre. Puis ensuite tu atteins un certain âge et lu te rends compte que, dans certains cas, tu te serais battu bien en vain. Alors tu te dis, bon, puisque je me serais battu bien en vain. pourquoi est-ce que je me battrais dans l'avenir contre ce que je sais ne pouvoir changer? Et cela te donne une certaine sérénité.

R.B. L'attitude du narrateur du Dernier Havre par rapport à celle d'Agaguk?

Y.T. Oui, si tu veux. Contre la mort, tu ne peux rien, tu peux prendre des pilules, ca fait le temps que ça fait, ensuite la mort va arriver quand même. Tu n'y peux rien, tu ne peux pas te battre contre cela; $\theta$ t méme si tu te battais, tu vas finir en révolté. tu vas mourir en... et ça ne te donnera rien, ators tu ne vas pas te battre pour rien.

A.B. L'absence de permanence...

Y.T. Tu te penses invulnérable, personne n'a réussi à te faire comprendre que tu étais vulnérable. Tu pars de là et ça devient un leitmotiv: seule la force est permanente, seule la puissance est permanente, seule la résistance physique poussée à l'extrême peut avoir de la valeur... Mais vraiment, les deux livres qui reflètent absolument mon état d'esprit actuel, c'est te Dernier Havre el Moi, Pierre Huneau; lis ces deux livres et oppose-les à ceux qui précèdent; tu es obligè de te dire que Thériault ne voit plus les choses de la même façon. Quand je parle de Pierre Huneau, je pense surtout à ce qui vient, á la suite que je veux donner au premier tome, même si celui-ci est déjà serein. Au fond d'ailleurs, il y avail des prémonitions de tout cela dans Ashini; la santé d'Ashini ne diminuait pas, mais sa femme mourait. Et Ashini n'avait plus rien que lui-même et sa forêt.

A.B. Alors Ashini a eu sa grande idée qui est devenue son grand projet; il faut avoir des projets, commencer, recommencer quelque chose?

Y.T. Avoir quelque chose à améliorer, des projets. L'homme en général, l'homme qui arrive à se satisfaire d'une façon totale, toute sa vie, d'un seul lieu de vie, d'un seul endroit où il a tout fait, où il n'y a plus rien à faire, cet homme est rare. Si j'étais entré dans cette maison-ci et qu'il n'y aurait rien eu à faire dedans, qu'elle aurait été parfaite, je n'aurais pas aimé cela. De méme, j'aimerais aller vivre en Italie, à cause du climat mais peut-ètre alors m'ennuierais-je d'ici? Mais cela fait partie de l'ensemble des projets...

R.B. Le malaise vient au moment où on arrête? 
Y.T. Oui, ah oui. Acheter une maison qui serait parfaite, dans laquelle il n'y aurait rien à changer, eh bien il ne se passerait pas grand temps avant que je commence à $y$ changer des choses.

A.B. Il faut que tu la lasses ta maison...

Y.T. D'une certaine façon, la recreer, la reconstruire continuellement, la refaire. J'ai souvent révé de bâtir ma maison, et je me rends compte que je pourrais me bătir la maison parfaite. Mais alors, it laudrait que je la vende, parce qu'il faudrait que j'en batisse une autre...

... en ce sens, le métier óécrivain est, lui, vraiment parfait. Ca s'adonne que j'avais un talent naturel de conteur. et j"ai conté des contes. C'est ca.

Renald BEAUBE UQAA 